\title{
Detection of human metapneumovirus in infants with acute respiratory tract infection
}

\author{
IOANNIS N. MAMMAS ${ }^{1}$, CHRYSSIE KOUTSAFTIKI $^{1}$, ELISABETH NIKA ${ }^{2}$, FOTINI VAGIA ${ }^{2}$, \\ APOSTOLOS ZARAVINOS ${ }^{5}$, KOSTAS N. PRIFTIS ${ }^{3}$, ALIKI VOYATZI ${ }^{2}$, MARIA THEODORIDOU ${ }^{4}$, \\ NIKOLAOS MYRIOKEFALITAKIS ${ }^{1}$ and DEMETRIOS A. SPANDIDOS ${ }^{5}$
}

\begin{abstract}
${ }^{1}$ First Department of Paediatrics; Departments of ${ }^{2}$ Clinical Microbiology, and ${ }^{3}$ Allergy-Pneumonology, 'Penteli' Children's Hospital; ${ }^{4}$ First Department of Paediatrics, 'Aghia Sofia' Children's Hospital, University of Athens School of Medicine, Athens; ${ }^{5}$ Department of Clinical Virology, University of Crete School of Medicine, Heraklion, Crete, Greece
\end{abstract}

Received August 3, 2010; Accepted December 20, 2010

DOI: $10.3892 / \mathrm{mmr} .2011 .416$

\begin{abstract}
Both respiratory syncytial virus (RSV) and human metapneumovirus (hMPV), a recently discovered respiratory pathogen classified in the Paramyxoviridae family, have been associated with acute respiratory tract infections (ARTI) in infants. The aim of our study was to determine the incidence and clinical features of hMPV infection in infants presenting with ARTI at a tertiary children's hospital in Greece during the 2007-2008 and 2008-2009 winter seasons. Routine nasal swabs were obtained from 127 infants younger than 12 months of age. The specimens were tested for the presence of hMPV and RSV antigens using validated enzyme-linked immunoassays. The mean age of the studied infants was 3 months (range 19 days to 12 months). hMPV was detected in 8 (6.3\%) children, 4 boys and 4 girls, while RSV was detected in 39 (30.7\%) children, 22 boys and 17 girls. In 7 children, hMPV was identified as a unique viral pathogen, while only 1 child was co-infected with hMPV and RSV. Bronchiolitis was diagnosed in all hMPV-positive cases, while only two cases required hospitalization and supplemental oxygen therapy. Our results provide further evidence of the importance of hMPV as a pathogen associated with community-acquired ARTI in infants in Greece.
\end{abstract}

\section{Introduction}

The human metapneumovirus (hMPV) was first described in June 2001 (1) in children from the Netherlands, and has since been shown to be a common respiratory pathogen

Correspondence to: Dr Ioannis N. Mammas, First Department of Paediatrics, 'Penteli' Children's Hospital, Palaia Penteli, Athens 15236, Greece

E-mail: mammasjo@googlemail.com

Key words: human metapneumovirus, acute respiratory tract infection, infants, Greece presenting during childhood (1-3). hMPV is a negative singlestranded RNA virus of the Paramyxoviridae family and the Metapneumovirus genus, which shows a variable seasonal pattern affecting children more frequently during the winter and spring (4). Serological studies have shown that almost $90 \%$ of children have been exposed to hMPV before the age of 5 years (5).

The incidence of hMPV infections worldwide accounts for at least $5-10 \%$ of acute respiratory tract infections (ARTI) in hospitalized children and for at least 3\% of patients who visit their general practitioner for ARTI $(2,3)$. The seasonality of hMPV infections resembles that of respiratory syncytial virus (RSV) infection, with recurrent epidemics during the winter months (3). Although hMPV infection has clinical characteristics similar to that of RSV, the precise epidemiological and clinical features of hMPV infection in childhood have yet to be fully established.

To the best of our knowledge, data on the presence and clinical impact of hMPV infections in children with ARTI in Greece is limited. This study aimed to determine the epidemiological and clinical characteristics of hMPV infections among hospitalized and non-hospitalized young children with acute respiratory tract infections in Athens, Greece.

\section{Materials and methods}

Children presenting with ARTI at the Paediatric Emergency Department of the 'Penteli' Children's Hospital in Athens, Greece, during the 2007-2008 and 2008-2009 winter seasons were enrolled. After clinical examination of infants presenting with respiratory infections, nasal wash aspirate specimens were obtained using a sterile catheter. Specimens were maintained at $4^{\circ} \mathrm{C}$ and transferred to the Microbiology Laboratory at the 'Penteli' Children's Hospital in Athens on a daily basis. Specimens were obtained over a 24-month period encompassing two winter seasons.

The specimens were tested for the presence of hMPV antigens using the validated enzyme-linked immunoassay Biotrin hMPV EIA (6) as recommended by the manufacturer (Biotrin International Ltd., Dublin, Ireland). The hMPV EIA is an 
antigen capture assay for the qualitative detection of hMPV antigens in human respiratory specimens. RSV was detected using the TRU RSV test (Meridian Bioscience Europe, Italy). This is a rapid, qualitative, lateral-flow immunoassay for the detection of RSV antigens in human respiratory samples (7).

Case notes for children included in this study were reviewed and clinical data were collected. Statistical analysis was performed using SPSS software version 11.5. Demographic and clinical features were compared between hMPV/ RSV-infected and non-infected children using the $\chi^{2}$ and the Mann-Whitney U tests. The cut-off for statistical significance was set at $\mathrm{p}<0.05$.

\section{Results}

During the 24-month study period, 127 infants younger than 12 months of age with ARTI were enrolled for the detection of hMPV and RSV: 63 infants during the 2007-2008 winter period and 64 infants during the 2008-2009 winter period. In all cases, specimen collection was adequate for laboratory analysis of both viruses.

Sixty-three children did not require hospitalization, while 64 children were admitted. Among the hospitalized children, 55 were discharged with a diagnosis of bronchiolitis, 2 with upper respiratory tract infection, and 7 with pneumonia. Among non-hospitalized children, 42 had an upper respiratory tract infection, while 21 had bronchiolitis. Bronchiolitis was defined as an acute respiratory illness characterized by wheezing on physical examination, with no radiological evidence of pneumonia. Diagnosis of pneumonia was based on radiological evidence, while upper respiratory infection was characterized by the presence of rhinitis without auscultation findings on physical examination. The mean age of the infants included in our sample was 3 months (range 19 days to 12 months). Table I shows the demographic characteristics of the children included in our study.

hMPV was detected in 8 children, 4 boys and 4 girls, while RSV was detected in 39 children, 22 boys and 17 girls. The overall frequencies of hMPV and RSV infection were 6.3 and $30.7 \%$, respectively. During the first year of the study (2007-2008), hMPV was detected in $6(9.5 \%)$ children compared to only $2(3.1 \%)$ during the second year (2008-2009). The respective rates for RSV were 20/63 (31.7\%) and 19/64 (29.7\%). The months with the highest hMPV activity were February and March, while RSV was detected more frequently during the months of January and February. In 7 children, hMPV was identified as a unique viral pathogen, while only 1 child was co-infected with hMPV and RSV.

The demographic and clinical features, signs and symptoms of hMPV-positive ( $n=8)$ and RSV-positive $(n=39)$ children with ARTI included in our study are presented in Tables II and III. The median age of the hMPV-infected children was 3.5 months (range 20 days to 7 months), while the median age of the RSV-infected children was 5.5 months (range 35 days to 11 months). Bronchiolitis was diagnosed in all hMPV-positive cases, while among RSV-positive cases, bronchiolitis was diagnosed in $33 / 39$ (84.6\%), pneumonia in $1 / 39(2.6 \%)$ and upper respiratory tract infection in 5/39 (12.8\%) children. Among the hMPV-positive cases, coughing occurred at a rate
Table I. Demographic and clinical characteristics of children included in the study $(\mathrm{n}=127)$ and detection of hMPV and RSV infection.

\begin{tabular}{|c|c|}
\hline Characteristic & No. $(\%)$ \\
\hline \multicolumn{2}{|l|}{ Age } \\
\hline Median & 3 months \\
\hline Range & 19 days - 12 months \\
\hline \multicolumn{2}{|l|}{ Gender } \\
\hline Male & $69(54.3)$ \\
\hline Female & $58(45.7)$ \\
\hline \multicolumn{2}{|l|}{ Ethnicity } \\
\hline Greek & $73(57.5)$ \\
\hline Albanian & $47(37.0)$ \\
\hline Other & $7 \quad(5.5)$ \\
\hline \multicolumn{2}{|l|}{ Area } \\
\hline Urban & $105(82.7)$ \\
\hline Rural & $22(17.3)$ \\
\hline \multicolumn{2}{|l|}{ Winter period } \\
\hline 2007-2008 & $63(49.6)$ \\
\hline 2008-2009 & $64(50.4)$ \\
\hline \multicolumn{2}{|l|}{ Diagnosis } \\
\hline Upper ARTI & $44(34.6)$ \\
\hline Bronchiolitis & $76(59.8)$ \\
\hline Croup & $0 \quad(0)$ \\
\hline Asthma & $0 \quad(0)$ \\
\hline Pneumonia & $7 \quad(5.5)$ \\
\hline \multicolumn{2}{|l|}{ Hospitalization } \\
\hline Hospitalized & $64(50.4)$ \\
\hline Non-hospitalized & $63(49.6)$ \\
\hline \multicolumn{2}{|c|}{ Oxygen requirements } \\
\hline Yes & $51(40.2)$ \\
\hline No & $76(59.8)$ \\
\hline \multicolumn{2}{|l|}{ Virus detected } \\
\hline hMPV & $8 \quad(6.3)$ \\
\hline RSV & $39(30.7)$ \\
\hline
\end{tabular}

of 6/8 (75\%), similar to the rate of infection with RSV (30/39, $76.9 \%)$. Vomiting occurred less often in cases of hMPV than RSV infection $(1 / 8,12.5 \%$ vs. $11 / 39,28.2 \%)$. On physical examination, wheezing was noted more often in children with hMPV than in children with RSV infection $(8 / 8,100 \%$ vs. $34 / 39,87.2 \%$ ). Rhinitis was noted at a similar rate in both RSV- and hMPV-positive children $(3 / 8,37.5 \%$ vs. $15 / 39$, $38.5 \%)$. Two hMPV-positive (25\%) and 19 RSV-positive $(48.7 \%)$ children required hospitalization and supplemental oxygen therapy. The mean duration of hospitalization was 6.5 days among hMPV- or RSV-positive children and 4.5 days among hMPV/RSV-negative children $(\mathrm{p}=0.011)$. The means of the duration of supplemental oxygen therapy were 3.5 days for hMPV and 2 days for RSV ( $\mathrm{p}=0.025)$. RSV and hMPV 
Table II. Demographic and clinical features of hMPV-positive $(n=8)$ and RSV-positive $(n=39)$ children with ARTI.

\begin{tabular}{cc}
\hline hMPV-positive & RSV-positive \\
no. $(\%)$ & no. $(\%)$ \\
\hline
\end{tabular}

Age
Median
Range

Gender

$\begin{array}{lrr}\text { Male } & 4 & (50) \\ \text { Female } & 4 & (50) \\ \text { Ethnicity } & & \\ \text { Greek } & & \\ \text { Albanian } & 4 & (50) \\ \text { Other } & 4 & (50) \\ & 0 & (0)\end{array}$

Area

$$
\begin{aligned}
& \text { Urban } \\
& \text { Rural }
\end{aligned}
$$

\section{5 months}

20 days -7 months

5.5 months
35 days - 11 months

$22(56.4)$

17 (43.6)

$20(51.3)$

$18(46.2)$

1 (2.6)

$34(87.2)$

$5(12.8)$

\begin{tabular}{|c|c|c|}
\hline 2007-2008 & $6 \quad(75)$ & $20(51.3)$ \\
\hline 2008-2009 & $(25)$ & $19(48.7)$ \\
\hline
\end{tabular}

Winter period

Diagnosis

$\begin{array}{lrrrr}\text { Upper ARTI } & 0 & (0) & 5 & (12.8) \\ \text { Bronchiolitis } & 8 & (100) & 33 & (84.6) \\ \text { Croup } & 0 & (0) & 0 & (0) \\ \text { Asthma } & 0 & (0) & 0 & (0) \\ \text { Pneumonia } & 0 & (0) & 1 & (2.6)\end{array}$

Hospitalization

$\begin{array}{llll}\text { Hospitalized } & 2 & (25) & 19(48.7) \\ \text { Non-hospitalized } & 6 & (75) & 20(51.3)\end{array}$

Oxygen requirements

$\begin{array}{lllll}\text { Yes } & 2 & (25) & 19 & (48.7) \\ \text { No } & 6 & (75) & 20 & (51.3)\end{array}$

co-infection was detected in 1 child with bronchiolitis, who required hospitalization and supplemental oxygen therapy.

\section{Discussion}

In this study, the presence of hMPV was demonstrated in Greek infants with ARTI. hMPV was detected in 8 infants at a rate of $6.3 \%$. To our knowledge, this is the first study to report the incidence of hMPV infection in children with ARTI in Greece. Previously, Xepapadaki et al (11) studied 56 Greek children hospitalized with acute bronchiolitis. Investigators from other countries have described variable frequencies of hMPV infection in children with ARTI specimens ranging from 2.6 to $25.3 \%(4,8-10)$. Our findings suggest that hMPV is a significant cause of hospitalization, a finding which is consistent with the results from other studies (8).

Our results indicate a low incidence of hMPV infections in infants with ARTI in Greece during the winter period of
Table III. Clinical symptoms and signs in hMPV-positive $(\mathrm{n}=8)$ and RSV-positive $(n=39)$ children with ARTI.

\begin{tabular}{lrrrr}
\hline & $\begin{array}{c}\text { hMPV-positive } \\
\text { no. }(\%)\end{array}$ & \multicolumn{2}{c}{$\begin{array}{r}\text { RSV-positive } \\
\text { no. }(\%)\end{array}$} \\
\hline Cough & 6 & $(75)$ & 30 & $(76.9)$ \\
Fever & 1 & $(12.5)$ & 5 & $(28.2)$ \\
Irritability & 0 & $(0)$ & 2 & $(5.1)$ \\
Reduced feeding & 4 & $(50)$ & 28 & $(71.8)$ \\
Vomiting & 1 & $(12.5)$ & 11 & $(28.8)$ \\
Diarrhea & 0 & $(0)$ & 5 & $(28.2)$ \\
Rhinitis & 3 & $(37.5)$ & 15 & $(38.5)$ \\
Wheezing & 8 & $(100)$ & 34 & $(87.2)$ \\
Rhonchi & 0 & $(0)$ & 1 & $(2.6)$ \\
Pharynghitis & 0 & $(0)$ & 2 & $(5.1)$ \\
\hline
\end{tabular}

2008-2009. This finding is consistent with previous data showing that hMPV has both seasonal and annual distribution $(8,12-15)$. In a study by Caracciolo et al (13), the incidence of hMPV infection was $25.3 \%$ during the 2005-2006 winterspring season, whereas a much lower rate of infection $(4.7 \%)$ was found during the following season. In a study conducted among French children by Manoha et al (14), hMPV was detected in $10.1 \%$ of children during the 2002-2003 winter season and in 3.3\% during the 2003-2004 winter season. In the hMPV outbreak described by Dollner et al (15) in Norwegian children during the 2002-2003 winter season, hMPV was detected in $57 \%$ of children with respiratory tract infection during the months of November to January.

In our study, all hMPV-positive children, 2 hospitalized and 6 non-hospitalized, were diagnosed with bronchiolitis. hMPV has thus far been identified in children with either upper or lower respiratory tract infection or both, asthma exacerbation, croup, or influenza-like illness $(4,9,8,12,14-16)$. Bronchiolitis is the most common diagnosis in children identified as having hMPV infection, while upper respiratory infection, pneumonia and asthma exacerbations have been reported less frequently $(4,8,12-15)$. In the study by Dollner et al (15), upper respiratory tract infections and mild-to-severe bronchiolitis were most common, but a relatively high proportion of hospitalized children developed severe pneumonia. hMPV infection has a milder clinical course than RSV infection $(4,11)$. In a study by von Linstow et al (9), overall symptoms and clinical findings were similar among hMPV- and RSV-positive children, but more RSV-infected children required respiratory support.

In childhood, RSV is considered to be the most common pathogen of bronchiolitis, and is also identified in communityacquired pneumonia. However, in clinical practice, the cause in the majority of cases cannot be determined. hMPV has been proposed as the second most common pathogen after RSV in children younger than 3 years of age hospitalized with respiratory tract infections $(4,9,12)$. Our study showed that during the 2007-2008 winter season, hMPV activity peaked after the RSV season. On the other hand, during the 2008-2009 winter season, hMPV and RSV epidemics occurred simultaneously. 
Estrada et al (17) reported 5 children with severe hMPVassociated infection requiring admission to the Pediatric Intensive Care Unit and a mean duration of 34 days of treatment. In the study by Dollner et al (15), 14 children with hMPV-positive ARTI required supplemental oxygen, 1 was treated with continuous positive airway pressure and 2 were ventilated mechanically. In both studies, half of the children had an underlying chronic disease, including neurological disorders and asthma. In the study by Noyola et al (12), an underlying disorder was noted in $25.6 \%$ of hMPV-positive children, including prematurity, congenital heart disease, neurological disorders, malignancies and congenital malformations. In a study by Dare et al (18), hMPV proved to be a more frequent infection than adenovirus, RSV, influenza $A$ and $\mathrm{B}$ and paramyxoviruses 1-3 in lung transplant recipients with respiratory symptoms (19). In our study, none of the hMPVpositive patients were admitted to the Pediatric Intensive Care Unit. One hMPV-positive baby was born prematurely; however this did not play a significant role in the clinical picture or outcome of hMPV infection.

RSV and hMPV co-infection was detected in 1 child with bronchiolitis, who required hospitalization and supplemental oxygen therapy. RSV and hMPV co-infection has been detected in several studies $(4,8,13,14)$. In a study by Williams et al (4), the co-infection rate was $6 \%$ among hMPV-positive children, while other researchers $(10,13)$ have detected $\mathrm{hMPV/RSV}$ co-infection more frequently, at rates of up to $30.8 \%$. RSV/hMPV co-infection has been proposed as a risk factor for severe respiratory tract infection during childhood $(10,13)$. In the study by Caracciolo et al $(13)$, hMPV/RSV co-infected children developed pneumonia more frequently than hMPV infected children. In the study by Foulongne et al (10), the duration of hospitalization and the requirements for supplemental oxygen were increased in hMPV/RSV co-infected children compared to hMPV- or RSV-positive children. However, other investigators have noted no apparent difference in the clinical severity, duration of hospitalization or requirement of oxygen between children with co-infections and children infected with hMPV alone $(9,13)$. Further research is therefore required to investigate the possible role of hMPV/RSV co-infection in the pathogenesis of respiratory tract infection during childhood.

Despite the lower sensitivity of immunoassays compared to RT-PCR (20), the Biotrin hMPV EIA has been optimized as an immunoassay with excellent specificity (6). Recently, in the study by Kukavica-Ibrulj et al (6), hMPV EIA showed a sensitivity of $81 \%$, a specificity of $100 \%$, a positive predictive value of $100 \%$ and a negative predictive value of $77 \%$ compared to viral culture and RT-PCR. Moreover, the usage of fresh samples in our study increased the sensitivity of our method, as cycles of freeze and thaw lead to antigen degradation, affecting antigen detection. Detection of hMPV by ELISA is a rapid, non-expensive and sensitive laboratory method that can be introduced into clinical practice to detect hMPV in children with ARTI.

In conclusion, hMPV was detected in the respiratory secretions of $6.3 \%$ of infants under 12 months of age with ARTI. Our results provide further evidence of the importance of hRSV and hMPV as pathogens associated with communityacquired ARTI in infants. They also indicate a low incidence of hMPV infection in hospitalized infants in Greece during the 2008-2009 winter period compared to the 2007-2008 winter period. All our hMPV-positive patients had a mild clinical course. To the best of our knowledge, this is the first study to report the incidence of hMPV infections in infants with ARTI in Greece. The development of new molecular diagnostic tests may enable us to clarify the viral causes of acute respiratory tract infections in clinical practice.

\section{Acknowledgements}

We are indebted to all the staff of the Department of Microbiology at the 'Penteli' Children's Hospital for assistance in the collection of clinical specimens.

\section{References}

1. Van der Hoogen BG, van Doomun GJJ, Fockens JC, et al: Prevalence and clinical symptoms of human metapneumovirus infevtion in hospitalized patients. J Infect Dis 188: 1571-1517, 2003.

2. Kahn JS: Human metapneumovirus: a newly emerging respiratory pathogen. Curr Opin Infect Dis 16: 255-258, 2003.

3. Van den Hoogen BG, Osterhaus DM and Fouchier RA: Clinical impact and diagnosis of human metapneumovirus infection. Pediatr Infect Dis J 23: S25-S32, 2004.

4. William JV, Harris PA, Tollefson SJ, et al: Human metapneumovirus and lower respiratory tract disease in otherwise healthy infants and children. N Engl J Med 250: 443-450, 2004.

5. Leung J, Esper F, Weibel C and Kahn JS: Seroepidemioloy of human metapneumovirus (hMPV) on the basis of a novel enzyme-linked immunosorbent assay utilizing hMPV fusion protein expressed in recombinant vesicular stomatitis virus. J Clin Microbiol 43: 1213-1219, 2005.

6. Kukavica-Ibrulj I and Boivin G: Detection of human metapneumovirus antigens in nasopharyngeal aspirates using an enzyme immunoassay. J Clin Virol 44: 88-90, 2008.

7. Swenson PD and Kaplan MH: Rapid detection of respiratory syncytial virus in nasopharyngeal aspirates by a commercial immunoassay. J Clin Microbiol 23: 485-488, 1986.

8. Esper F, Boucher D, Weibel C, Martinello RA and Kahn JS: Human metapneumovirus infection in the United States: clinical manifestations associated with a newly emerging respiratory infection in children. Pediatrics 111: 1407-1410, 2003.

9. Von Linstow ML, Larsen HH, Eugen-Olsen J, et al: Human metapneumovirus and respiratory syncytial virus in hospitalized danish children with acute respiratory tract infection. Scand J Infect Dis 36: 578-584, 2004.

10. Foulongne V, Guyon G, Rodière $M$ and Segondy M: Human metapneumovirus infection in young children hospitalized with respiratory tract disease. Pediatr Infect Dis J 25: 354-359, 2006.

11. Xepapadaki P, Psarras S, Bossios A, et al: Human metapneumovirus as a causative agent of acute bronchiolitis in infants. J Clin Virol 30: 267-270, 2004.

12. Noyola DE, Alpuche-Solis AG, Herrera-Diaz A, Soria-Guerra RE and Sanchez-Alvarado J: Human metapneumovirus infections in Mexico: epidemiological and clinical characteristics. J Med Microbiol 54: 969-974, 2005.

13. Caracciolo S, Minini C, Colombrita D, et al: Human metapneumovirus infection in young children hospitalized with acute respiratory tract disease: virologic and clinical features. Pediatr Infect Dis J 27: 406-412, 2008.

14. Manoha C, Espinosa S, Aho SL, Huet F and Pothier P: Epidemiological and clinical features of hMPV, RSV and RVs infections in young children. J Clin Virol 38: 221-226, 2007.

15. Dollner H, Risnes K, Radtke A and Nordbo SA: Outbreak of human metapneumovirus infection in Norwegian children. Pediatr Infect Dis J 23: 436-440, 2004.

16. Stockton J, Stephenson I, Fleming D and Zambon M: Human metapneumovirus as a cause of community-acquired respiratory illness. Emerg Infect Dis 8: 897-901, 2002.

17. Estrada B, Carter M, Barik S, et al: Severe human metapneumovirus infection in hospitalized children. Clin Pediatrics 46: 258-262, 2007. 
18. Dare R, Sanghavi S, Bullota A, et al: Detection of human metapneumovirus (hMPV) infection in immunosuppressed lung transplant recipients and children evaluated for pertussis. J Clin Microbiolol 45: 548-552, 2007.

19. Larcher $\mathrm{C}$, Geltner $\mathrm{C}$, Fischer H, et al: Human metapneumovirus infection in lung transplant recipients: clinical presentation and epidemiology. J Heart Lung Transplant 24: 1891-1901, 2005.
20. Hopkins MJ, Redmond C, Shaw JM, Hart IJ and Hart A: Detection and characterization of human matapneumovirus from children with acute respiratory symptoms in north-west England, UK. J Clin Virol 42: 273-279, 2008. 